\title{
"CHOLESTEROGENIC" DIETARY FACTORS AND MILK AND MILK FAT YIELDS IN DAIRY COWS
}

\author{
Pellervo SaARinen \\ Department of Animal Husbandry, University of Helsinki
}

Received November 15, 1958

In an earlier publication, "Diet and plasma cholesterol in dairy cows" (5), it was established that there is a statistically verifiable positive partial correlation between the cholesterol level in the blood of the cow and certain dietary factors. The most important of these factors, in addition to the energy requirements of the animal, seem to be the digestible crude fat and fibre present in the food ration. However, the same kind of effect can also be produced by obesity of the animal and in certain cases even by temporary overfeeding.

As the liver is the only organ supplying significant amounts of cholesterol to the blood plasma and therefore is the actual source of plasma cholesterol, it was supposed that the grade of plasma cholesterol may possibly be used as a reliable indicator for some factors which affect the normal liver functions, but which are otherwisc hard to establish (5).

In addition, from the point of view of animal nutrition it would be of prime importance to know to what extent these cholesterogenic dietary factors result in strain on the liver and/or other metabolic organs, or whether they reduce the stress.

To throw light on this latter group of questions we have tried at this institute to complete the picture given by blood plasma cholesterol measurements, at the same time determining the quantity of 17-ketosteroids segregated in the urine. The effect of ACTH and cortisone injections on the level of blood cholesterol has also been examined. According to the results obtained, variations in the function of the pituitary-cortical system do not have any particular effect on the level of plasma cholesterol in the blood, or vice versa. Investigations which are at present being carried out on simultaneous variations in thyroid function have as yet not given any results in which the picture given by the cholesterol measurements can be made fundamentally clearer under normal conditions. As a fourth possibility, an attempt has been made to use the variations in the level of milk yield in explaining possible effects from strain. 
In addition to the partial correlation between the dietary factors and the level of blood plasma cholesterol in the cow, mentioned at the beginning of this paper, the author has reported earlier that a positive partial correlation also exists between the milk or milkd fat yields and the level of blood plasma cholesterol in the cow (4). In view of this, it is possible that the effect of the cholesterogenic dietary factors can be verified directly from variations in milk or milk fat yields. Statistical investigations have thus been made to examine whether the existing cholesterogenic factors studied in earlier investigations have any specific effects on the milk or milk fat yields.

\section{Experimental procedure}

Investigations have been made on the dietary factors observed in an earlier investigation as probably having some kind of effect on the level of blood plasma cholesterol (5).

The material for analysis was obtained in two different ways. First, calculations were carried out using the experimental results obtained from 12 feeding tests $(5$, trials I-XII) made to investigate the effect of diet on the level of blood cholesterol. These results were then checked separately, using a second series of experimental results obtained from feeding trials (trials XIII-XXVII) carried out at the Department of Animal Husbandry, Agricultural Research Centre, for other purposes and also suitable for this purpose. Only results from normally fertilized animals were taken in these feeding trials. The trials had been carried out on estates where there was some certainty that the cows had been fed approximately to standard for several years in succession, and that they had normally received sufficient energy, protein and minerals from their food.

All the trials had been carried out during a period of indoor feeding, using the individual feeding method. Trials I-XII comprised several experimental periods of a comparatively short duration, only about two weeks in length. Trials XIIIXXVII were of longer duration, lasting about four months. They consisted of three 30-day experimental periods, to which were added additional 10-day change-over periods.

Eliminating the effects of the stage of lactation and variations in nutritional requirements, the level of yield $\left(\mathrm{x}_{1}\right)$ was measured as a relative deviation from each individual normal lactation curve for each experimental period. For the same reason an attempt was made to express the dietary factors as far as possible as quantities independent of the stage of lactation.

For use in comparison, curves for the normal yields of the cows were plotted for the year in which the short-period tests had been performed (trials I--XII), as well as for the previous year and the year following, i.e., on the basis of the average yields for three successive years. Further, an attempt was made to eliminate the temporary effects of possible diseases and nutritional disturbances. For the longer period feeding trials the normal curves were plotted only on the basis of average yields for the year preceding and the year following the year of the tests. 


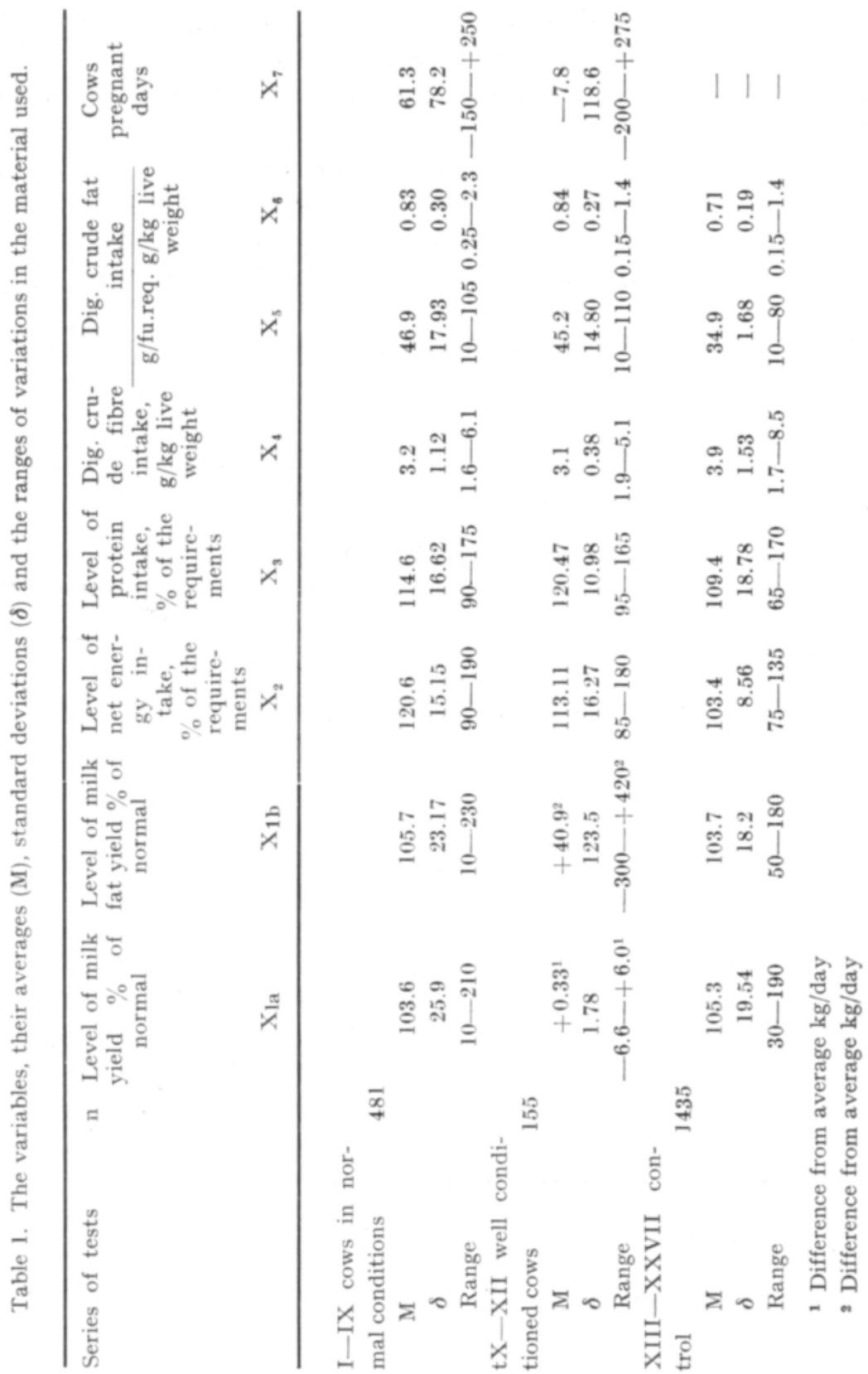


The momentary milk and fat yields obtained from the experimental feedings were calculated on the basis of the average values for 10-day periods in each experimental period.

The relative milk yield was determined by calculating what percentage the milk yield in the experimental feeding was of the yield according to the normal lactation curve for the corresponding period of time after calving.

Otherwise the experimental and analytical methods of calculation were the same as in previous investigations (5).

The investigation material, the different variables, their average values and ranges of variation are presented in greater detail in Table 1.

As Table 1 shows, on an average the level of net energy and digestible crude protein intake was somewhat above the requirements of the animals, but a few cases of slight under-nourishment were included in the material.

In trials I-XII the net energy intake varied from $85-190 \%$ of the standard requirements, and the digestible crude protein intake varied from $90-175 \%$. In trials XIII-XXVII it was originally attempted to keep to the same lower limits. However, in some individual cases, the corresponding lower limits in some of the feeding trials in this series were lower than these, being $75 \%$ in the case of net energy and $65 \%$ in the case of digestible crude protein.

\section{Results}

First the results were calculated on the basis of the actual deviation values (kg/day). The combinations of the results from each series are presented in Table 2 in the first and last rows of the figures. As appears from the figures in the last column of this table, a slight negative effect of the lactation stage and or of pregnancy $\left(\mathrm{x}_{7}\right)$ on the yield could still be statistically verifiable. On closer examination, this was observed to be due, at least in part, to the fact that the variations are generally

Table 2. Coefficients of partial correlation when the actual difference in milk or milk fat yield $\left(\mathrm{X}_{1}=\right.$ differences from normal $\mathrm{kg} / \mathrm{day}$ ) is considered as the dependent variable and the level of net energy intake $\left(\mathrm{X}_{2}=\%\right.$ of the requirements), the level of dig. crude protein intake $\left(\mathrm{X}_{3}=\%\right.$ of the requirements), the dig. crude fibre intake $\left(X_{4}=g / k g\right.$ live weight $)$, the dig. crude fat intake $\left(X_{5}=g /\right.$ Sc. feed unit required and $X_{6}=g / k g$ live weight), and the stage of lactation $\left(X_{7}=\right.$ cows pregnant days $)$ as independent variables. Trials I-IX. Normally fertilized cows in normal condition.

\footnotetext{
$\mathrm{X}_{2}=$ level of net $\mathrm{X}_{3}=$ level of $\mathrm{X}_{4}=$ dig. crude $\mathrm{X}_{5}=$ dig. crude $\mathrm{X}_{6}=$ dig. crude $\mathrm{X}_{7}=$ cows pregenergy intake protein intake fibre intake fat intake $\mathrm{g} / \mathrm{fu}$. fat intake $\mathrm{g} / \mathrm{kg}$ nant days req. live weight

Actual difference in milk yield

$\mathrm{r}_{12 ; 34567}=-0.1599 \quad \mathrm{r}_{13 ; 24567}=+0.0454 \quad \mathrm{r}_{14: 23567}=+0.1193 \quad \mathrm{r}_{15 ; 23467}=-0.3054 \quad \mathrm{r}_{16 ; 23457}=+0.4657 \quad \mathrm{r}_{17 ; 23456}=-0.1311$

Relative difference in millk yield

$\mathrm{r}_{12 ; 34567}=-0.1923 \quad \mathrm{r}_{13 ; 24567}=+0.0382 \quad \mathrm{r}_{14 ; 23567}=+0.0493 \quad \mathrm{r}_{15 ; 23467}=-0.3627 \quad \mathrm{r}_{16 ; 23457}=+0.3568 \quad \mathrm{r}_{17 ; 23456}=+0.0649$

Actual difference in milk fat yield

$r_{12 ; 34567}=-0.2022 \quad r_{13 ; 24567}=+0.0900 \quad r_{14: 23567}=+0.0123 \quad r_{15 ; 23467}=-0.4220 \quad r_{16 ; 23457}=+0.4017 \quad r_{17 ; 23456}=+0.0672$
} 
greater during a stage of high yield than during a stage of low yield. If on the other hand, the variations of yield were, measured as percentages of the corresponding level of yield in the normal curve, such an effect no longer seemed to occur, as the last column in the second row of figures in Table 2 shows.

For this reason only the coefficients of the partial correlation calculated on the basis of the relative yield values were included in the following tables.

A similar attempt was also made to express the dietary factors as variables independent of the stage and level of yield. In this respect only the levels of energy and protein intake $\left(\mathrm{x}_{2}\right.$ and $\left.\mathrm{x}_{3}\right)$ could be determined to entire satisfaction. The crude fibre intake was not quite so easily determinable as an independent quantity. In satisfying the nutritional requirements of the animal by making the concentration ratio of the food ration at the same time such that undisturbed utilisation of the food was possible, the crude fibre content of the food ration, calculated either as percentage dry matter weight, in grams per feed unit, or in grams per kilogram live weight, varied according to the stage of yield. However, the last-mentioned quantity was least dependent on the level of yield in the experimental material and generally in practice, too. Because of this, the crude fibre intake was measured in grams per kilo live weight in the accompanying calculations. For the same reason, the digestible crude fat intake was measured in grams per feed unit. However, as the experimental results showed a more significant positive partial correlation between the digestible crude fat intake, measured in $\mathrm{g} / \mathrm{kg}$ live weight $\left(\mathrm{x}_{6}\right)$, and the relative level of yield

Table 3. Coefficients of partial correlation when the relative milk yield $\left(\mathrm{X}_{1}=\operatorname{milk}\right.$ yield $\%$ of normal) is considered as the dependent variable and the level of net energy intake $\left(\mathrm{X}_{2}=\%\right.$ of the requirements), the level of dig. crude protein intake $\left(X_{3}=\%\right.$ of the requirements), the dig. crude fibre intake $\left(X_{4}=\right.$ $\mathrm{g} / \mathrm{kg}$ live weight) and the dig. crude fat intake $\left(\mathrm{X}_{5}=\mathrm{g} / \mathrm{Sc}\right.$. feed unit required and $\mathrm{X}_{6}=\mathrm{g} / \mathrm{kg}$ live weight) as independent variables.

Trials I-IX. Normally fertilized cows in normal condition $(n=481)$.

$\begin{array}{ccc}\mathrm{X}_{2}=\text { level of net } & \mathrm{X}_{3}=\text { level of } & \mathrm{X}_{4}=\text { dig. crude } \\ \text { energy intake } & \text { protein intake } & \text { fibre intake }\end{array}$

$\mathrm{X}_{5}=$ dig. crude fat intake $\mathrm{g} / \mathrm{fu}$. reg.
$\mathrm{X}_{6}=$ dig. crude fat intake $\mathrm{g} / \mathrm{kg}$ live weight

$\begin{array}{llllllll}\mathrm{r}_{12}=-0.4517 & \mathrm{r}_{13}=-0.2809 & \mathrm{r}_{14}=+0.2679 & \mathrm{r}_{15}=-0.2962 & \mathrm{r}_{16} & =+0.0419 \\ \mathrm{r}_{12 ; 3}=-0.3729 & \mathrm{r}_{13 ; 2}=+0.0607 & \mathrm{r}_{14 ; 2}=+0.1980 & \mathrm{r}_{15 ; 2}=-0.1195 & \mathrm{r}_{16 ; 2}=+0.1374 \\ \mathrm{r}_{12 ; 4}=-0.4198 & \mathrm{r}_{13 ; 4}=-0.2479 & \mathrm{r}_{14 ; 3}=+0.2327 & \mathrm{r}_{15 ; 3}=-0.1995 & \mathrm{r}_{16 ; 3}=+0.1347 \\ \mathrm{r}_{12 ; 5}=-0.3741 & \mathrm{r}_{13 ; 5}=-0.1746 & \mathrm{r}_{14 ; 5}=+0.2256 & \mathrm{r}_{15 ; 4}=-0.2593 & \mathrm{r}_{16 ; 4}=+0.0379 \\ \mathrm{r}_{12 ; 6}=-0.4666 & \mathrm{r}_{13 ; 6}=-0.3067 & \mathrm{r}_{14 ; 6}=+0.2674 & \mathrm{r}_{15 ; 6}=-0.5858 & \mathrm{r}_{16 ; 5}=+0.5304 \\ \mathrm{r}_{12 ; 34}=-0.4180 & \mathrm{r}_{13 ; 24}=+0.0131 & \mathrm{r}_{14 ; 23}=+0.2004 & \mathrm{r}_{15 ; 23}=-0.1350 & \mathrm{r}_{16 ; 23}=+0.1267 \\ \mathrm{r}_{12 ; 35}=-0.3460 & \mathrm{r}_{13 ; 25}=+0.0320 & \mathrm{r}_{14 ; 25}=+0.1873 & \mathrm{r}_{15 ; 24}=-0.1002 & \mathrm{r}_{16 ; 24}=+0.1285 \\ \mathrm{r}_{12 ; 36}=-0.3704 & \mathrm{r}_{13 ; 26}=+0.0286 & \mathrm{r}_{14 ; 26}=+0.1921 & \mathrm{r}_{15 ; 26}=-0.4486 & \mathrm{r}_{16 ; 25}=+0.4528 \\ \mathrm{r}_{12 ; 45}=-0.3546 & \mathrm{r}_{13 ; 45}=-0.1574 & \mathrm{r}_{14 ; 35}=+0.2128 & \mathrm{r}_{15 ; 34}=-0.1755 & \mathrm{r}_{16 ; 34}=+0.1211 \\ \mathrm{r}_{12 ; 46}=-0.4342 & \mathrm{r}_{13 ; 46}=-0.2719 & \mathrm{r}_{14 ; 36}=+0.2254 & \mathrm{r}_{15 ; 36}=-0.5332 & \mathrm{r}_{16 ; 35}=+0.5180 \\ \mathrm{r}_{12 ; 56}=-0.2206 & \mathrm{r}_{13 ; 56}=-0.1153 & \mathrm{r}_{14 ; 56}=+0.0698 & \mathrm{r}_{15 ; 46}=-0.5441 & \mathrm{r}_{16 ; 45}=+0.4965 \\ \mathrm{r}_{12 ; 345}=-0.3977 & \mathrm{r}_{13 ; 245}=+0.0343 & \mathrm{r}_{14 ; 235}=+0.2289 & \mathrm{r}_{15 ; 234}=-0.1166 & \mathrm{r}_{16 ; 234}=+0.1155 \\ \mathrm{r}_{12 ; 346}=-0.3537 & \mathrm{r}_{13 ; 246}=+0.0384 & \mathrm{r}_{14 ; 236}=+0.1937 & \mathrm{r}_{15 ; 236}=-0.4489 & \mathrm{r}_{16 ; 235}=+0.4469 \\ \mathrm{r}_{12 ; 356}=-0.1922 & \mathrm{r}_{13 ; 256}=+0.0286 & \mathrm{r}_{14 ; 256}=+0.0656 & \mathrm{r}_{15 ; 246}=-0.4242 & \mathrm{r}_{16 ; 245}=+0.4242 \\ \mathrm{r}_{12 ; 456}=-0.2193 & \mathrm{r}_{13 ; 456}=-0.1118 & \mathrm{r}_{14 ; 356}=+0.0642 & \mathrm{r}_{15 ; 34}=-0.4991 & \mathrm{r}_{16 ; 345}=+0.5433 \\ \mathrm{r}_{12 ; 3456}=-0.1933 & \mathrm{r}_{13 ; 2456}=+0.0355 & \mathrm{r}_{14 ; 2356}=+0.0673 & \mathrm{r}_{15 ; 234}=-0.4172 & \mathrm{r}_{16 ; 2345}=+0.4167\end{array}$


Table 4. Coefficients of partial correlation when the relative milk fat yield $\left(\mathrm{X}_{1}=\right.$ milk fat $\%$ of normal) is considered as the dependent variable, and the level of net energy intake $\left(\mathrm{X}_{2}=\%\right.$ of the requirements), the level of dig crude protein intake $\left(\mathrm{X}_{3}=\%\right.$ of the requirements), the dig. crude fibre intake $\left(\mathrm{X}_{4}=\right.$ $\mathrm{g} / \mathrm{kg}$ live weight) and the dig. crude fat intake $\left(X_{5}=g / S c\right.$. feed unit required and $X_{6}=g / k g$ live weight) as independent variables.

Trials I-IX. Normally fertilized cows in normal condition $(\mathrm{n}=\mathbf{4 8 1})$.

\begin{tabular}{|c|c|c|c|c|}
\hline $\begin{array}{l}\mathrm{X}_{2}=\text { level of net } \\
\text { energy intake }\end{array}$ & $\begin{array}{l}\mathrm{X}_{3}=\text { level of } \\
\text { protein intake }\end{array}$ & $\begin{array}{c}\mathrm{X}_{4}=\text { dig. crude } \\
\text { fibre intake }\end{array}$ & $\begin{array}{c}\mathrm{X}_{5}=\text { dig. crude } \\
\text { fat intake } \mathrm{g} / \mathrm{fu} . \\
\text { req. }\end{array}$ & $\begin{array}{l}\mathrm{X}_{6}=\text { dig. crude } \\
\text { fat intake } \mathrm{g} / \mathrm{kg} \\
\text { live weight }\end{array}$ \\
\hline
\end{tabular}

\begin{tabular}{|c|c|c|c|c|c|c|c|c|c|}
\hline$r_{12}$ & $=-0.4905$ & $r_{13}$ & $=-0.2489$ & $\mathrm{r}_{14}$ & $=+0.2205$ & $r_{15}$ & $=-0.3415$ & $\mathrm{r}_{16}$ & $=+0.0102$ \\
\hline$r_{12 ; 3}$ & $=-0.4591$ & $\mathrm{r}_{13 ; 2}$ & $=+0.1585$ & $r_{14: 2}$ & $=+0.1376$ & $r_{15 ; 2}$ & $=-0.1583$ & $r_{16 ; 2}$ & $=+0.1117$ \\
\hline $\mathrm{r}_{12 ; 4}$ & $=-0.4657$ & $r_{13 ; 4}$ & $=-0.2199$ & $r_{14 ; 3}$ & $=+0.1866$ & $\mathrm{r}_{15 ; 3}$ & $=-0.2662$ & $r_{16 ; 3}$ & $=+0.0894$ \\
\hline$r_{12 ; 5}$ & $=-0.4023$ & $\mathrm{r}_{13 ; 5}$ & $=-0.1156$ & $\mathrm{r}_{14 ; 5}$ & $=+0.1684$ & $\mathrm{r}_{15 ; 4}$ & $=-0.3128$ & $r_{16 ; 4}$ & $=+0.0058$ \\
\hline$r_{12 ; 6}$ & $=-0.5001$ & $\mathrm{r}_{13 ; 6}$ & $=-0.2634$ & $\mathrm{r}_{14: 6}$ & $=+0.2204$ & $\mathrm{r}_{15 ; 6}$ & $=-0.6192$ & $\mathrm{r}_{16: 5}$ & $=+0.5495$ \\
\hline${ }{ }_{12 ; 34}$ & $=-0.4464$ & $r_{13 ; 24}$ & $=-0.1643$ & $\mathrm{r}_{14: 23}$ & $=+0.1444$ & $r_{15 ; 23}$ & $=-0.1973$ & $r_{16: 23}$ & $=+0.0766$ \\
\hline$r_{12 ; 35}$ & $=-0.4285$ & $r_{13: 25}$ & $=+0.1975$ & $r_{14 ; 25}$ & $=+0.1224$ & $r_{15 ; 24}$ & $=-0.1454$ & $\mathrm{r}_{16: 24}$ & $=+0$ \\
\hline $\mathrm{r}_{12 ; 36}$ & $=-0.4572$ & $\mathrm{r}_{13: 26}$ & $=+0.1364$ & $\mathrm{r}_{14 ; 26}$ & $=+0.1321$ & $\mathrm{r}_{15 ; 26}$ & $=-0.4795$ & $\mathrm{r}_{16: 25}$ & $=+$ \\
\hline $\mathrm{r}_{12 ; 45}$ & $=-0.3876$ & $\mathrm{r}_{13 ; 45}$ & $=-0.1011$ & $\mathrm{r}_{14 ; 35}$ & $=+0.1589$ & $r_{15 ; 34}$ & $=-0.2483$ & $r_{16: 34}$ & $=+0$. \\
\hline$r_{12 ; 46}$ & $=-0.4748$ & $\mathrm{r}_{13 ; 46}$ & $=-0.2324$ & $r_{14: 36}$ & $=+0.1812$ & $r_{15: 36}$ & $=-0.5819$ & $r_{16: 35}$ & $=+0.5422$ \\
\hline$r_{12 ; 56}$ & $=-0.2511$ & $\mathrm{r}_{13 ; 56}$ & $=-0.0422$ & $\mathrm{r}_{14 ; 56}$ & $=+0.0092$ & $r_{15 ; 46}$ & $=-0.5933$ & $r_{16 ; 45}$ & $=+0.5305$ \\
\hline $\mathrm{r}_{12 ; 345}$ & $=-0.4194$ & $r_{13: 245}$ & $=-0.1392$ & $\mathrm{r}_{14 ; 235}$ & $=+0.1265$ & $\mathrm{r}_{15 ; 234}$ & $=-0.1849$ & $r_{16 ; 234}$ & $=+0.0676$ \\
\hline $\mathrm{r}_{12 ; 346}$ & $=-0.4451$ & $r_{13 ; 246}$ & $=+0.1440$ & $\mathrm{r}_{14 ; 236}$ & $=+0.1398$ & $r_{15 ; 236}$ & $=-0.4840$ & $r_{16: 235}$ & $=+0.4559$ \\
\hline $\mathrm{r}_{12 ; 356}$ & $=-0.2898$ & $\mathrm{r}_{13 ; 256}$ & $=+0.1554$ & $\mathrm{r}_{14 ; 256}$ & $=-0.0161$ & $r_{15: 246}$ & $=-0.4653$ & $r_{16: 245}$ & $=+0.4568$ \\
\hline $\mathrm{r}_{12 ; 456}$ & $=-0.2512$ & $\mathrm{r}_{13 ; 456}$ & $=-0.0425$ & $r_{14 ; 356}$ & $=-0.0114$ & $r_{15 ; 346}$ & $=-0.5623$ & $r_{16: 345}$ & $=+0.5252$ \\
\hline $\mathrm{r}_{12 ; 3456}$ & $=-0.2897$ & $\mathrm{r}_{13: 2456}$ & $=+0.1549$ & $\mathrm{r}_{14 ; 2356}$ & $=-0.0088$ & $\mathrm{r}_{15 ; 2346}$ & $=-0.4680$ & $r_{16: 2345}$ & \\
\hline
\end{tabular}

Table 5. Coefficients of partial correlation when the actual difference in milk fat yield $\left(\mathrm{X}_{1}=\right.$ difference from normal $\mathrm{g} /$ day $)$ is considered as the dependent variable and the level of net energy intake $\left(\mathrm{X}_{2}=\right.$ $\%$ of the requirements), the level of dig. crude protein intake $\left(\mathrm{X}_{\mathrm{a}}=\%\right.$ of the requirements), the dig. crude fibre intake $\left(X_{4}=g / k g\right.$ live weight) and the crude fat intake $\left(X_{5}=g / S c\right.$. feed unit required and $\mathrm{X}_{6}=\mathrm{g} / \mathrm{kg}$ live weight) as independent variables.

Trials $\mathrm{X}$-XII. Well conditioned normally fertilized cows $(\mathrm{n}=155)$.

\begin{tabular}{|c|c|c|c|c|}
\hline $\begin{array}{c}\mathrm{X}_{2}=\text { level of net } \\
\text { energy intake }\end{array}$ & $\begin{array}{l}\mathrm{X}_{3}=\text { level of } \\
\text { protein intake }\end{array}$ & $\begin{array}{c}\mathrm{X}_{4}=\text { dig. crude } \\
\text { fibre intake }\end{array}$ & $\begin{array}{c}\mathrm{X}_{\mathrm{s}}=\text { dig. crude } \\
\text { fat intake } \mathrm{g} / \mathrm{fu} . \\
\text { req. }\end{array}$ & $\begin{array}{l}\mathrm{X}_{6}=\text { dig. crude } \\
\text { fat intake } \mathrm{g} / \mathrm{kg} \\
\text { live weight }\end{array}$ \\
\hline
\end{tabular}

\begin{tabular}{|c|c|c|c|c|c|c|c|c|c|}
\hline$r_{12}$ & $=-0.4115$ & $\mathrm{r}_{13}$ & $=-0.3778$ & $\mathrm{r}_{14}$ & $=+0.3926$ & $r_{15}$ & $=-0.3012$ & $\mathrm{r}_{16}$ & $=+0.0773$ \\
\hline$r_{12 ; 3}$ & $=-0.3430$ & $r_{13 ; 2}$ & $=-0.2989$ & $\mathrm{r}_{14 ; 2}$ & $=+0.2950$ & $r_{15 ; 2}$ & $=-0.1983$ & $r_{16: 2}$ & $=-0.0249$ \\
\hline $\mathrm{r}_{12 ; 4}$ & $=-0.3217$ & $\mathrm{r}_{13 ; 4}$ & $=-0.3397$ & $\mathrm{r}_{14 ; 3}$ & $=+0.3566$ & $\mathrm{r}_{15 ; 3}$ & $=-0.1849$ & $\mathrm{r}_{16}$ & $=+0.1300$ \\
\hline $12 ; 5$ & $=-0.3498$ & $r_{13 ; 5}$ & $=-0.2991$ & $r_{14: 5}$ & $=+0.34 .54$ & $\mathrm{r}_{15 ; 4}$ & $=-0.2309$ & $\mathrm{r}_{16 ; 4}$ & $=-0.0286$ \\
\hline $12 ; 6$ & $=-0.4061$ & $\mathrm{r}_{13 ; 6}$ & $=-0.3901$ & $\mathrm{r}_{14 ; 6}$ & $=+0.3870$ & $\mathrm{r}_{15 ; 6}$ & $=-0.5143$ & $r_{16: 5}$ & $=+0$. \\
\hline $12 ; 34$ & $=-0.2631$ & $\mathrm{r}_{13: 24}$ & $=-0.2856$ & $\mathrm{r}_{14 ; 23}$ & $=+0.2814$ & $\mathrm{r}_{15 ; 23}$ & $=-0.1144$ & $r_{16: 23}$ & $=+0.0352$ \\
\hline $12: 35$ & $=-0.3136$ & $r_{13: 25}$ & $=-0.2540$ & $r_{14 ; 25}$ & $=+0.2734$ & $\mathrm{r}_{15: 24}$ & $=-0.1625$ & $\mathrm{r}_{16: 24}$ & $=-0.0886$ \\
\hline $\mathrm{r}_{12 ; 36}$ & $=-0.3219$ & $\mathrm{r}_{13: 26}$ & $=-0.2999$ & $\mathrm{r}_{14 ; 26}$ & $=+0.3060$ & $\mathrm{r}_{15: 26}$ & $=-0.3496$ & $\mathrm{r}_{16: 25}$ & $=+0.2949$ \\
\hline$r_{12 ; 45}$ & $=-0.2793$ & $\mathrm{r}_{13 ; 45}$ & $=-0.2841$ & $\mathrm{r}_{14 ; 35}$ & $=+0.3329$ & $\mathrm{r}_{15 ; 34}$ & $=-0.1270$ & $r_{16: 34}$ & $=+0.0304$ \\
\hline$r_{12 ; 46}$ & $=-0.3313$ & $r_{13 ; 46}$ & $=-0.3399$ & $r_{14 ; 36}$ & $=+0.3361$ & $\mathrm{r}_{15 ; 36}$ & $=-0.4170$ & $\mathrm{r}_{16: 35}$ & $=+0.3989$ \\
\hline $\mathrm{r}_{12 ; 56}$ & $=-0.0574$ & $r_{13 ; 56}$ & $=-0.2184$ & $r_{14 ; 56}$ & $=+0.0869$ & $r_{15 ; 46}$ & $=-0.3761$ & $r_{16 ; 45}$ & $=+0.3069$ \\
\hline$r_{12 ; 345}$ & $=-0.2455$ & $\mathrm{r}_{13: 245}$ & $=-0.2510$ & $r_{14 ; 235}$ & $=+0.2706$ & $r_{15: 234}$ & $=-0.0818$ & $r_{16: 234}$ & $=-0.0285$ \\
\hline$r_{12 ; 346}$ & $=-0.2628$ & $r_{13 ; 246}$ & $=-0.2740$ & $\mathrm{r}_{14: 236}$ & $=+0.2807$ & $r_{15: 236}$ & $=-0.2847$ & $r_{16: 235}$ & $=+0.2646$ \\
\hline$r_{12 ; 356}$ & $=-0.0549$ & $r_{13 ; 256}$ & $=-0.2176$ & $\mathrm{r}_{14 ; 256}$ & $=+0.1111$ & $r_{15 ; 246}$ & $=-0.2085$ & $\mathrm{r}_{16 ; 245}$ & $=+0.1585$ \\
\hline$r_{12 ; 456}$ & $=-0.0900$ & $\mathrm{r}_{13 ; 456}$ & $=-0.2272$ & $\mathrm{r}_{14 ; 356}$ & $=+0.1080$ & $r_{15 ; 346}$ & $=-0.2820$ & $r_{16 ; 345}$ & $=+0.2559$ \\
\hline$r_{12 ; 3456}$ & $=-0.0948$ & $r_{13 ; 2456}$ & $=-0.2291$ & $\mathrm{r}_{14 ; 2356}$ & $=+0.1330$ & $\mathrm{r}_{15 ; 2346}$ & $=-0.1418$ & $r_{16: 2345}$ & \\
\hline
\end{tabular}


than could be explained on the basis of the partial correlation between the stage of yield (days since calving) and the fat content of the food ration, the first-mentioned factor $\left(\mathbf{x}_{6}\right)$ was treated specially as a separate independent variable.

Tables 3 and 4 present results from the effects of different dietary factors on the level of the relative milk and milk fat yields in the short-period feeding tests on cows in normal condition. In these statistics the effect of the level of net energy intake $\left(x_{2}\right)$ appeared slightly negative throughout. In slight protein over-nourishment no detrimental effects on the level of yield appeared in these animals in normal conditions. The statistical effect of digestible crude fibre intake $\left(\mathrm{x}_{4}\right)$, insomuch as it appeared, was positive. The effect of the digestible crude fat intake again appeared negative as regards the amount in grams.

For comparison, Table 5 presents results from a small material from trials $\mathrm{X}$-XII, calculated on the basis of the actual differences in milk yield in wellconditioned cows. These results were very uniform with the results in the previous Tables 3 and 4 . However, in the case of wellconditioned obese cows, it should be noted that the excessive intake of protein was statistically in negative partial correlation with the level of milk yield.

Tables 6 and 7 present corresponding results from the long-period feeding trials carried out at the Department of Animal Husbandry, Agricultural Research Centre. In this material the effects appeared somewhat slighter, but approximately just as significant statistically as in the shortperiod tests mentioned earlier. This indicated

Table 6. Coefficients of partial correlation when the relative milk yield $\left(\mathrm{X}_{1}=\right.$ milk $\%$ of normal) is considered as the dependent variable and the level of net energy intake $\left(\mathrm{X}_{2}=\%\right.$ of requirements), the level of dig. crude protein intake $\left(X_{3}=\%\right.$ of the requirements), the dig. crude fibre intake $\left(X_{4}=g / k g\right.$ live weight), and the dig. crude fat intake $\left(X_{5}=g / S c\right.$. feed unit required and $X_{6}=g / k g$ live weight) as independent variables.

Trials XII-XXVII. The feeding tests of the Department of Animal Husbandry $(\mathrm{n}=1435)$.

\begin{tabular}{|c|c|c|c|c|}
\hline $\begin{array}{c}\mathrm{X}_{2}=\text { level of net } \\
\text { energy intake }\end{array}$ & $\begin{array}{l}\mathrm{X}_{3}=\text { level of } \\
\text { protein intake }\end{array}$ & $\begin{array}{l}\mathrm{X}_{4}=\text { dig. crude } \\
\text { fibre intake }\end{array}$ & $\begin{array}{c}\mathrm{X}_{5}=\text { dig. crude } \\
\text { fat intake } \mathrm{g} / \mathrm{fu} . \\
\text { req. }\end{array}$ & $\begin{array}{c}\mathrm{X}_{6}=\text { dig. crude } \\
\text { fat intake } \mathrm{g} / \mathrm{kg} \\
\text { live weight }\end{array}$ \\
\hline
\end{tabular}

$\begin{array}{llll}r_{12}=+0.0697 & r_{13}=+0.0333 & r_{14}=+0.2050 \\ r_{12 ; 3}=+0.0630 & r_{13 ; 2}=+0.0146 & r_{14 ; 2}=+0.1954 \\ r_{12 ; 4}=+0.0291 & r_{13 ; 4}=+0.0373 & r_{14 ; 3}=+0.2056 \\ r_{12 ; 5}=-0.0448 & r_{13 ; 5}=-0.0568 & r_{14 ; 5}=+0.1031 \\ r_{12 ; 6}=+0.0208 & r_{13 ; 6}=-0.0017 & r_{14 ; 6}=+0.1742 \\ r_{12 ; 34}=+0.0193 & r_{13 ; 24}=+0.0303 & r_{14 ; 23}=+0.1971 \\ r_{12 ; 35}=-0.0330 & r_{13 ; 25}=-0.0479 & r_{14 ; 25}=+0.1096 \\ r_{12 ; 36}=+0.0218 & r_{13 ; 26}=-0.0064 & r_{14 ; 26}=+0.1731 \\ r_{12 ; 45}=-0.0580 & r_{13 ; 45}=-0.0475 & r_{14 ; 35}=+0.0982 \\ r_{12 ; 46}=-0.0035 & r_{13 ; 46}=+0.0112 & r_{14 ; 36}=+0.1746 \\ r_{12 ; 56}=-0.0738 & r_{13 ; 56}=-0.0717 & r_{14 ; 56}=+0.1025 \\ r_{12 ; 345}=-0.0482 & r_{13 ; 245}=-0.0345 & r_{14 ; 235}=+0.1044 \\ r_{12 ; 346}=-0.0062 & r_{13 ; 246}=+0.0124 & r_{14 ; 236}=+0.1734 \\ r_{12 ; 356}=-0.0090 & r_{13 ; 256}=-0.0411 & r_{14 ; 256}=+0.1144 \\ r_{12 ; 456}=-0.0872 & r_{13 ; 456}=-0.0624 & r_{14 ; 356}=+0.1034 \\ r_{12 ; 3456}=-0.0245 & r_{13 ; 2456}=-0.0275 & r_{14 ; 2356}=+0.1061\end{array}$

$r_{15}=+0.1580$
$r_{15 ; 2}=+0.1436$
$r_{15 ; 3}=+0.1545$
$r_{15 ; 4}=+0.1144$
$r_{15 ; 6}=-0.1822$
$r_{15 ; 23}=+0.1430$
$r_{15 ; 24}=+0.1107$
$r_{15 ; 26}=-0.1779$
$r_{15 ; 34}=+0.1088$
$r_{15 ; 36}=-0.1791$
$r_{15 ; 46}=-0.1845$
$r_{15 ; 234}=+0.1072$
$r_{15 ; 236}=-0.1761$
$r_{15: 246}=-0.1784$
$r_{15: 346}=-0.1819$
$r_{15: 2346}=-0.1771$

$\mathrm{r}_{16}=+0.3644$

$\mathrm{r}_{16: 2}=+0.3610$

$\mathrm{r}_{16: 3}=+0.3670$

$\mathrm{r}_{16 ; 4}=+0.3230$

$r_{16: 5}=+0.3744$

$\mathrm{r}_{16: 23}=+0.3635$

$\mathrm{r}_{16: 24}=+0.3266$

$\mathrm{r}_{16: 25}=+0.3743$

$\mathrm{r}_{16: 34}=+0.3243$

$r_{16: 35}=+0.3768$

$r_{16: 45}=+0.3512$

$\mathrm{r}_{16: 234}=+0.3269$

$r_{16: 235}=+0.3761$

$\mathrm{r}_{16: 245}=+0.3527$

$\mathrm{r}_{16: 345}=+0.3527$

$\mathrm{r}_{16: 2345}=+0.3533$ 
Table 7. Coefficients of partial correlation when the relative milk fat yield $\left(\mathrm{X}_{1}=\right.$ milk fat $\%$ of normal) is considered as the dependent variable and the level of net energy intake $\left(\mathrm{X}_{2}=\%\right.$ of the requirements), the level of dig. crude protein intake $\left(\mathrm{X}_{3}=\%\right.$ of the requirements), the dig. crude fibre intake $\left(\mathrm{X}_{4}=\right.$ $\mathrm{g} / \mathrm{kg}$ live weight), and the dig. crude fat intake $\left(\mathrm{X}_{5}=\mathrm{g} / \mathrm{Sc}\right.$. feed unit required and $\mathrm{X}_{6}=\mathrm{g} / \mathrm{kg}$ live weight) as independent variables.

Trials XIII-XXVII. The feeding tests of the Department of Animal Husbandry $(\mathrm{n}=1435)$.

\begin{tabular}{|c|c|c|c|c|}
\hline $\begin{array}{c}\mathrm{X}_{2}=\text { level of net } \\
\text { energy intake }\end{array}$ & $\begin{array}{l}\mathrm{X}_{3}=\text { level of } \\
\text { protein intake }\end{array}$ & $\begin{array}{c}\mathrm{X}_{4}=\text { dig. crude } \\
\text { fibre intake }\end{array}$ & $\begin{array}{c}\mathrm{X}_{5}=\text { dig. crude } \\
\text { fat intake } \mathrm{g} / \mathrm{fu} . \\
\text { req. }\end{array}$ & $\begin{array}{c}\mathrm{X}_{6}=\text { dig. crude } \\
\text { fat intake } \mathrm{g} / \mathrm{kg} \\
\text { live weight }\end{array}$ \\
\hline
\end{tabular}

\begin{tabular}{|c|c|c|c|c|c|c|c|c|c|}
\hline $\mathrm{r}_{12}$ & $=+0.0923$ & $\mathrm{r}_{13}$ & $=+0.0444$ & $\mathrm{r}_{14}$ & $=+0.2775$ & $\mathrm{r}_{15}$ & $=+0.0407$ & $\mathrm{r}_{16}$ & $=+0.2879$ \\
\hline $\mathrm{r}_{12 ; 3}$ & $=+0.0833$ & $\mathrm{r}_{13 ; 2}$ & $=+0.0196$ & $\mathrm{r}_{14 ; 2}$ & $=+0.2649$ & $\mathrm{r}_{15 ; 2}$ & $=+0.0119$ & $\mathrm{r}_{16: 2}$ & $=+0.2735$ \\
\hline $\mathrm{r}_{12 ; 4}$ & $=+0.0373$ & $\mathrm{r}_{13 ; 4}$ & $=+0.0498$ & $\mathrm{r}_{14 ; 3}$ & $=+0.2784$ & $\mathrm{r}_{15 ; 3}$ & $=+0.0317$ & $\mathrm{r}_{16 ; 3}$ & $=+0.2856$ \\
\hline$r_{12 ; 5}$ & $=+0.0065$ & $\mathrm{r}_{13 ; 5}$ & $=-0.0248$ & $\mathrm{r}_{14 ; 5}$ & $=+0.2121$ & $\mathrm{r}_{15 ; 4}$ & $=-0.0274$ & $\mathrm{r}_{16 ; 4}$ & $=+0.2164$ \\
\hline $\mathrm{r}_{12 ; 6}$ & $=+0.0848$ & $\mathrm{r}_{13 ; 6}$ & $=+0.0368$ & $\mathrm{r}_{14 ; 6}$ & $=+0.2793$ & $\mathrm{r}_{15 ; 6}$ & $=-0.2792$ & $r_{16 ; 5}$ & $=+0.3908$ \\
\hline$r_{12 ; 34}$ & $=+0.0245$ & $\mathrm{r}_{13 ; 24}$ & $=+0.0415$ & $\mathrm{r}_{14 ; 23}$ & $=+0.2673$ & $\mathrm{r}_{15 ; 23}$ & $=+0.0091$ & $\mathrm{r}_{16 ; 23}$ & $=+0.2740$ \\
\hline $\mathrm{r}_{12 ; 35}$ & $=+0.0120$ & $\mathrm{r}_{13 ; 25}$ & $=-0.0261$ & $\mathrm{r}_{14 ; 25}$ & $=+0.2066$ & $\mathrm{r}_{15 ; 24}$ & $=-0.0403$ & $\mathrm{r}_{16 ; 24}$ & $=+0.2178$ \\
\hline $\mathrm{r}_{12 ; 36}$ & $=+0.0776$ & $\mathrm{r}_{13 ; 26}$ & $=+0.0181$ & $\mathrm{r}_{14 ; 26}$ & $=+0.2674$ & $\mathrm{r}_{15 ; 26}$ & $=-0.2753$ & $r_{16 ; 25}$ & $=+0.3806$ \\
\hline $\mathrm{r}_{12 ; 45}$ & $=-0.0198$ & $r_{13 ; 45}$ & $=-0.0047$ & $\mathrm{r}_{14: 35}$ & $=+0.2048$ & $\mathrm{r}_{15 ; 34}$ & $=-0.0408$ & $\mathrm{r}_{16 ; 34}$ & $=+0.2149$ \\
\hline $\mathrm{r}_{12 ; 46}$ & $=+0.0470$ & $\mathrm{r}_{13 ; 46}$ & $=+0.0577$ & $\mathrm{r}_{14 ; 36}$ & $=+0.2795$ & $\mathrm{r}_{15 ; 36}$ & $=-0.2706$ & $r_{16 ; 35}$ & $=+0.3846$ \\
\hline $\mathrm{r}_{12 ; 56}$ & $=+0.0503$ & $\mathrm{r}_{13 ; 56}$ & $=-0.0044$ & $\mathrm{r}_{14: 56}$ & $=+0.2246$ & $\mathrm{r}_{15 ; 46}$ & $=-0.2885$ & $r_{16 ; 45}$ & $=+0.3437$ \\
\hline$r_{12 ; 345}$ & $=-0.0187$ & $\mathrm{r}_{13 ; 245}$ & $=+0.0001$ & $\mathrm{r}_{14 ; 235}$ & $=+0.2051$ & $r_{15 ; 234}$ & $=-0.0479$ & $\mathrm{r}_{16 ; 234}$ & $=+0.2140$ \\
\hline $\mathrm{r}_{12 ; 346}$ & $=+0.0350$ & $\mathrm{r}_{13 ; 246}$ & $=+0.0488$ & $\mathrm{r}_{14 ; 236}$ & $=+0.2711$ & $\mathrm{r}_{15 ; 236}$ & $=-0.2745$ & $\mathrm{r}_{16 ; 235}$ & $=+0.3803$ \\
\hline $\mathrm{r}_{12 ; 356}$ & $=+0.0510$ & $r_{13 ; 256}$ & $=-0.0151$ & $\mathrm{r}_{14 ; 256}$ & $=+0.2134$ & $\mathrm{r}_{15 ; 246}$ & $=-0.2803$ & $r_{16: 245}$ & $=+0.3474$ \\
\hline $\mathrm{r}_{12 ; 456}$ & $=+0.0241$ & $r_{13 ; 456}$ & $=+0.0179$ & $r_{14 ; 356}$ & $=+0.2183$ & $r_{15 ; 346}$ & $=-0.2806$ & $\mathrm{r}_{16 ; 345}$ & $=+0.3462$ \\
\hline$r_{12 ; 3456}$ & $=+0.0198$ & $\mathrm{r}_{13 ; 2456}$ & $=+0.0123$ & $\mathrm{r}_{14: 2356}$ & $=+0.2057$ & $\mathrm{r}_{15 ; 2346}$ & $=-0.2804$ & $\mathrm{r}_{16 ; 2345}$ & $=+0.3447$ \\
\hline
\end{tabular}

some kind of adaptation to feeding in the long-period tests. Detrimental effects from an excessive intake of energy and protein could not be observed in these statistics. However, it should be noted that in this series of tests the excesses were relatively slight even in the most extreme cases (level of net energy intake $75-135 \%$ and level of protein intake $65-170 \%$ of the standards) and, on the other hand, under-nourishment could also be a limiting factor on the yield in certain cases. A rise in the level of yield was not obtained by heavier feeding, however, even under these circumstances.

\section{Discussion}

The statistical results obtained seem to indicate that the excessive intake of energy in the experiments described has had only some unmeasurable or negative effects on the level of milk and milk fat yields. In this respect the results are uniform with results obtained in Norway and Denmark using other experimental methods $(1,6)$. In the negative cases the effect of over-nourishment seems to appear somewhat more clearly in the level of milk and milk fat yields than in the blood plasma cholesterol content (5). This specifically indicates strain effects. The effects of excessive intake of protein seem to be similar in the level of plasma cholesterol and in the level of milk and milk fat yields. Increasing the proportion of digestible crude fibre in the food ration seems in certain cases to raise the level of milk and milk 
fat yields somewhat. This rise does not, however, entirely correspond to a simultaneous rise in the level of blood plasma cholesterol. The negative results obtained with the relative values of the digestible crude fat intake, calculated per feed unit, and the positive results with the simultaneous values in grams indicate that the amount of fat required by a dairy cow in its food varies during the different stages of yield. Thus the optimal level of digestible crude fat intake for the average stage of yield can be insufficient in stages of heavy milk yield, and again detrimentally large in stages of small yields.

\section{Summary and Conclusions}

The following results have been obtained from a statistical investigation carried out on experimental results from 27 feeding trials in which the effect of cholesterogenic dietary factors, observed in an earlier investigation (5), on the relative levels of milk and milk fat yields are compared.

An execessive intake of energy either has no statistically clear effect on the level of yield, or the effect is negative. In this respect the results obtained are uniform with experimental results obtained in Norway and Denmark using other methods $(1,6)$. In the negative cases the effect appears more clearly in the level of milk and milk fat yields than in the plasma cholesterol content.

An excessive intake of protein shows a negative effect only in well-conditioned obese cows.

An increase of the proportion of digestible crude fibre in the food ration appears in most cases to have an increasing effect on the milk yield. This effect, however, seems to be slighter than the effect on the blood plasma cholesterol content.

The level of milk and milk fat yields has proved to be statistically in positive partial correlation to the digestible crude fat intake in $\mathrm{g} / \mathrm{kg}$ live weight , and in negative partial correlation to the relative fat intake compared with the nutritional requirements of the animal. This indicates that the favourable level of fat intake varies according to the level of milk yield, being larger in stages of higher yields than in stages of small yields.

\section{REFERENCES}

(1) Hvidsten, H. 1951. Kraftforforbruk og forstyrke i mjolkeproduksjonen. Buskap og Avdratt, 3 , p. $3-7$.

(2) Jurila, VAPPU 1956. Aivolisäkkeen etulohkon ja lisämunuaisen kuorikerroksen toimintoihin liittyvistä rasitusilmiöistä kotieläimillä. Masters thesis.

(3) Lönneerg, Giтha 1954. Om inverkan av fodrets fett och protein pâ blodets kolesterol och urinens 17-ketosteroidhalt. Laudaturbeskrivning.

(4) SAARINEN, P. 1944. Lehmän veriplasman lipoidiaineosien vaikutuksesta maitorasvan muodostukseen. Ref. Uber den Einfluss einiger Lipoidbestandteile im Blutplasma der Kuh auf die Milchfetterzeugung. Acta Agralia Fennica, 57, 2, p. 1-131. 
(5) SaArinen, 1955. Diet and plasma cholesterol in dairy cows. Maataloustieteellinen Aikakauskirja (J. Sci. Agr. Soc. Finland), 27, p. 117-141.

(6) Wenzel Eskedal, H. \& Hansen, K. 1951. Forsøg med fodringens indflydelse på køernes holdbarhed. Oversigt. Forsogslaboratoriet 1950-1951. Ref.: Problems of animal feeding in Europe, p. 67-99, Published by FAO and EAAP, Rome 1955.

S E L OS T U S :

\title{
KOLESTEROGENISEN RAVINNON VAIKUTUS MAIDON JA MAITORASVAN TUOTOKSEEN
}

\author{
Pellervo SaArinen \\ Helsingin yliopiston kotieläintieteen laitos.
}

Kun 27 ruokintakokeesta saadun kỏetulosmateriaalin nojalla suoritettiin tilastollinen tutkimus, jossa verrattiin aikaisemmassa tutkimuksessa (5) kolesterogenisiksi havaittujen dieettisten tekijöiden vaikutusta lypsylehmien suhteelliseen maidon ja maitorasvan tuotostasoon, niin saatiin seuraavat tulokset.

Energiayliruokinnalla ei ole ollut joko mitään tilastollisesti selvää vaikutusta tuotostasoon tai tämä vaikutus on ollut negatiivinen. Tässä suhteessa saatu tulos on yhdenmukainen Norjassa ja Tanskassa muilla menetelmillä saatujen koetulosten kanssa $(1,6)$. Negatiivisissa tapauksissa vaikutus on esiintynyt maidon ja maitorasvan tuotostasossa selvempänä kuin plasman kolesterolipitoisuudessa.

Valkuaisvliruokinnalla ilmeni negatiivista vaikutusta vain voimakkaasti kunnostetuilla lehmillä.

Sulavan raakakuidun osuuden lisäämisellä rehuannoksessa näytti useimmissa tapauksissa olevan maidon tuotosta lisäävä vaikutus. Tämä vaikutus näyttää kuitenkin ilmenevän lievempänä kuin vastaava vaikutus plasman kolesterolipitoisuuteen.

Sulavan raakarasvan määrä rehuannoksessa grammoissa elopainokiloa kohden mitattuna on osoittautunut olevan tilastollisesti positiivisessa vuorosuhteessa ja eläinten ravinnontarpeeseen verrattu suhteellinen raakarasvan määrä taas negatiivisessa vuorosuhteessa maidon ja maitorasvan tuotostasoon. Tämä viittaa siihen, että edullisin rasvan määrä rehussa vaihtelee tuotoksen määrästä riippuen, ollen se suurempi runsaan tuotannon kuin vähäisen tuotanon vaiheessa. 